\title{
Enhanced spontaneous emission in semiconductor nanocrystal solids using resonant energy transfer for integrated devices
}

\author{
Sedat Nizamoglu1,1,34 and Hilmi Volkan Demir ${ }^{1,2,3,4}$ \\ Department of Electrical and Electronics Engineering ${ }^{1}$, Department of Physics ${ }^{2}$, Nanotechnology Research Center ${ }^{3}$ and Institute of \\ Materials Science and Nanotechnology ${ }^{4}$, Bilkent University, Ankara, 06800 Turkey \\ Tel:[+90](312) 290-1021 E-mail: volkan@bilkent.edu.tr
}

Size-tuneable optical properties of semiconductor nanocrystal (NC) quantum dots make them attractive for a wide range of device applications [1-10]. However, in these device applications, nanocrystals typically suffer from relatively low quantum efficiency (QE) when they are cast into solid form [11]. To reduce the effect of this problem, we propose and demonstrate the enhancement of spontaneous emission in nanocrystal solids by recycling their trapped excitons through resonant nonradiative Förster energy transfer (ET) for hybrid integrated devices. For this purpose, we designed closely packed CdSe/ZnS core/shell nanocrystal emitters with an energy gradient of approximately $160 \mathrm{meV}$ integrated on LEDs. In such a designed mixture of nanocrystal solids, the small quantum dots behaved as donors and the large quantum dots as acceptors, allowing for strong ET from the small ones to the large ones. Consequently, we obtained an emission enhancement of $46 \%$ for the large dots in the mixed energy gradient structure with respect to the luminescence of only large dots. This energy gradient structure led to a $13.2 \%$ increase in the quantum efficiency of the large dots in the presence of the small dots compared to the large dots alone as a result of the recycled excitons. We investigated the Förster energy transfer facilitated in these nanocrystal solid samples using both steady state and time resolved spectroscopy. As an integrated device application, we also demonstrated the hybrid use of these nanocrystal solids hybridized on near-UV InGaN/GaN LED for white light generation. The emission enhancement based on such carefully designed, cascaded energy transfer systems is critically important especially for white light emitting diodes in solid state lighting application, which makes one of the next solid state frontiers.

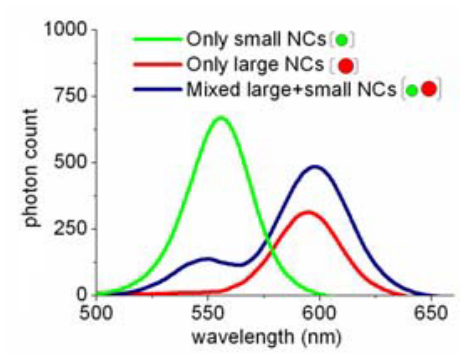

(a)

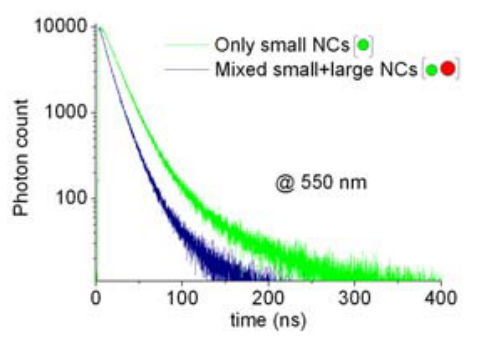

(b)

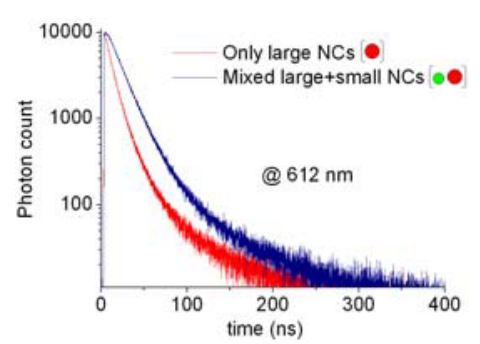

(c)

Figure 1. (a) Steady state photoluminescence spectra of only large nanocrystal solids (with a diameter of $8.2 \mathrm{~nm}$ ), only small nanocrystal solids (with a diameter of $7.7 \mathrm{~nm}$ ) and mixed large-small nanocrystal solids (with diameters of 8.2 and $7.7 \mathrm{~nm}$, respectively), (b) time resolved photoluminescence spectra of only small nanocrystal solids $(7.7 \mathrm{~nm})$ and mixed large-small nanocrystal solids $(8.2$ and $7.7 \mathrm{~nm}$ ) at $550 \mathrm{~nm}$, and (c) time resolved photoluminescence spectra of only large nanocrystal solids $(8.2 \mathrm{~nm})$ and mixed large-small nanocrystal solids $(8.2$ and $7.7 \mathrm{~nm})$ at $612 \mathrm{~nm}$.

Förster energy transfer is the nonradiative transfer of excitation energy from an excited molecule (donor) to a ground-state molecule (acceptor) [12]. To facilitate ET in nanocrystal solids, we used two types of CdSe/ZnS core/shell nanocrystals, one emitting at $540 \mathrm{~nm}$ and the other at $580 \mathrm{~nm}$ in solution, designed with diameters of $7.7 \mathrm{~nm}$ and $8.2 \mathrm{~nm}$, respectively [13]. For control groups, we prepared two test solid films that consisted of only small quantum dots and only large quantum dots separately. For nonradiative Förster energy transfer, we prepared mixed solid assembly of small and large nanocrystals and make their closely packed films containing exactly the same amount of only small and only large quantum dots as in the control group samples.

To study ET in steady state photoluminescence measurements, we used a Xenon halogen lamp in a monochromator as the excitation light source with an integrating sphere for collecting the total emission from the nanocrystal solids. Figure 1 (a) shows the photoluminescence spectrum of the mixed large-small nanocrystal solids along with those of only small and only large nanocrystal solids. In the mixed large-small nanocrystal solids, the emission peak of donors at $550 \mathrm{~nm}$ was quenched with respect to only small nanocrystal solids due to transferring their excitation energy to acceptors. On the other hand, the peak of acceptor emission at $612 \mathrm{~nm}$ was increased because of the excitation energy transferred from donors. Also because of the resonant nonradiative energy transfer, a relative red shift in the emission of large dots was observed in the mixed sample.

To study kinetics of the energy transfer in our nanocrystal solid samples, we performed time resolved spectroscopy measurements using a FluoTime 200 spectrometer (PicoQuant) and a time-correlated single photon 
counting (TCSPC) system of PicoHarp 300 with a calibrated time resolution of 4 ps. Figure 1 (b) shows time resolved photoluminescence spectroscopy of only small nanocrystal solids and that of energy gradient sample at $550 \mathrm{~nm}$, while Figure 1 (c) depicts the time resolved spectroscopy of only large nanocrystal solids and that of the energy gradient sample at $612 \mathrm{~nm}$, for all of which the pulsed excitation was at the center wavelength of $375 \mathrm{~nm}$. In Figure 1(b), the lifetime of the large-small nanocrystal solids at $550 \mathrm{~nm}$ was decreased with respect to the only small dots (because the donor small dots quenched due to transferring their excitation energy to the acceptor large dots). In contrast, in Figure 1(c), the lifetime of the large-small nanocrystal solids at $612 \mathrm{~nm}$ was increased with respect to the only large nanocrystal solids (because the acceptor large dots took the transferred energy from the donor small dots).

In the mixed nanocrystal solid film, the Förster energy transfer rate was found to be $2.86 \mathrm{~ns}^{-1}$. Here we observed an emission enhancement of $46 \%$ for the large dots in the mixed sample with respect to the only large dots and measured QE of the large dots in the mixed sample to be 0.291, which was 13.2 percent larger than it would be if there were no energy transfer. Here the confined excitons made spontaneous emission as usual; but, additionally, the portion of trapped excitons recycled by Förster energy transfer also contributed to the spontaneous emission, resulting in the enhancement of QE. We employed this assembly of large-small nanocrystal solids that exhibits strong Förster energy transfer also for white light generation. We used the same amount of nanocrystals in the mixed solid film as color converters enhanced with nonradiative energy transfer on an InGaN/GaN n-UV LED emitting at $383 \mathrm{~nm}$. In Figure 2, the emission spectra of the resulting integrated hybrid device are shown when it is electrically driven at various current injection levels at room temperature. The emission spectrum of this hybrid LED led to $(\mathrm{x}, \mathrm{y})$ tristimulus coordinates of $(0.44,0.40)$ and correlated color temperature (CCT) of $2872 \mathrm{~K}$.

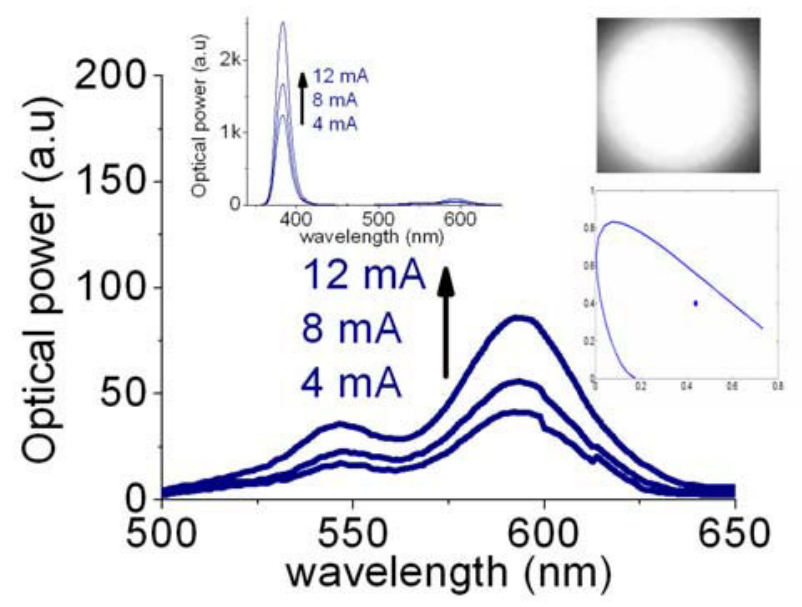

Figure 2. Emission spectra of energy gradient large-small CdSe/ZnS core/shell nanocrystal solids (with diameters of 8.2 and $7.7 \mathrm{~nm}$ ) hybridized on near-UV InGaN/GaN light emitting diode driven at different levels of current injection at room temperature, along with the corresponding $(x, y)$ coordinates and pictures of the hybrid LED while generating white light.

In conclusion, using resonant nonradiative Förster energy transfer, we demonstrated the spontaneous emission enhancement of $\mathrm{CdSe} / \mathrm{ZnS}$ core/shell nanocrystal solids integrated on near-UV InGaN/GaN light emitting diode for white light generation. As revealed both by their steady state and time resolved spectroscopy measurements, we observed a relative quantum efficiency enhancement for the acceptor nanocrystal quantum dots in the energy gradient structure by comparing with the monodisperse phase. As a result of the nonradiative energy transfer facilitated in the energy gradient structure, recycling trapped excitons into nanocrystal quantum dots led to the enhancement of quantum efficiency.

Acknowledgements: This work is supported by EU Network of Excellence PHOREMOST 511616 and Marie Curie IRG MOON 021391 and TUBITAK under the Project No. EEEAG 106E020, 104E114, 107E297, 107E088, 105E065, and 105E066. HVD also acknowledges additional support from ESF-EURYI and TUBA-GEBIP.

References

[1] R. C. Somers et al., Chem. Soc. Rev. 36, 579, 2007.

[2] V. Klimov et al., Science, 290, 314, 2000.

[3] H. Chen et al, IEEE Photon. Technol. Lett., 18, 1430, 2006.

[4] I. M. Soganci et al, Opt. Exp., 15, 14289, 2007.

[5] S. Nizamoglu et al., Nanotechnology, 18, 065709, 2007.

[6] S. Nizamoglu et al., Appl. Phys. Lett., 92, 031102, 2008.

[7] H. V. Demir et al., New J. of Phys., 9, 362, 2007.
[8] S. Nizamoglu et al., J. Opt. A: Pure Appl. Opt., 9, S419, 2007.

[9] S. Nizamoglu et al., Nanotechnology, 18, 405702, 2007.

[10] M. Ali et al., Nanotechnology, 18, 075401, 2007.

[11] A. Chistyakov et al., Laser Phys., 16, 1625, 2006.

[12] T. A. Klar et al., Adv. Mat., 17, 769, 2005.

[13] S. Nizamoglu et al., 2008 (submitted). 